\title{
Use of a side effect as a covariate in a problem of sequential analysis
}

\author{
Martha Bilotti-Aliaga \\ Department of Statistics, University of Michigan, Am Arbor, MI, USA
}

Received 14 April 1989; revised manuscript received 20 September 1991

Recommended by T.L. Lai

Abstract: A drug is administered sequentially to incoming patients. A response $Y$ to treatment and a covariate $X$ is measured ( $X$ might be a side effect). The experiment is stopped when the covariate falls outside some acceptable region. We study the effect that this optional stopping has on the significance level of the test and we found that this effect is surprisingly small in the examples considered. An approximation to the problem is found. This approximation does not depend on the distribution of the variable $X$ but only on the correlation coefficient between $X$ and $Y$.

AMS Subject Classification: Primary $62 \mathrm{~L} 10$.

Key words and phrases. Estimation; clinical trials; backward induction; dynamic programming; binary binomial case; testing without batches; testing with batches; Wiener process.

\section{Introduction and summary}

Suppose that there is an infinite sequence $\left(X_{i}, Y_{i}\right), i=1,2, \ldots$, of covariateresponse pairs which are independent with common unknown distribution $H$, and that the parameter $E(Y)=\theta$ is of primary interest. Let $F_{n}=\sigma\left\{X_{1}, \ldots, X_{n}\right\}$ denote the smallest $\sigma$-algebra with respect to which $X_{1}, \ldots, X_{n}$ are mcasurable. We sample sequentially according to a stopping rule $t$ depending on the $X_{i}$ 's.

Suppose that a one-sided hypothesis about $\theta$ is of interest, say $\mathrm{H}_{0}: \theta \leqslant \theta_{0}$, and that $\mathrm{H}_{0}$ is to be rejected if

$$
\sqrt{n}\left(\bar{Y}_{n}-\theta_{0}\right) / \hat{\sigma}_{n}>c
$$

at the time $t=n$ of stopping, where $\bar{Y}_{n}$ is the sample mean, $\hat{\sigma}_{n}^{2}, n \geqslant 1$, denotes a consistent sequence of estimates of the variance of $Y$, and $c$ is a constant. If we stop at time $t$, then the actual significance level is

Correspondence to: Dr. Martha Bilotti-Aliaga, Dept. of Statistics, 1444 Mason Hall, University of Michigan, Ann Arbor, MI 48109-1027, USA. 


$$
\alpha_{t}=P_{0}\left\{\frac{\sqrt{t}\left(\bar{Y}_{t}-\theta_{0}\right)}{\hat{\sigma}_{t}}>c\right\},
$$

where $P_{0}$ denotes probability when $\theta=\theta_{0}$ for fixed values of any nuisance parameters.

We may determine the maximum possible effect of such optional stopping on the attained significance level as follows. Let

$$
Z_{n}=P_{0}\left\{\frac{\sqrt{n}\left(\bar{Y}_{n}-\theta_{0}\right)}{\hat{\sigma}_{n}}>c \mid X_{1}, \ldots, X_{n}\right\}
$$

Then

$$
\alpha_{t}=E_{0}\left(Z_{t}\right)
$$

so we may determine the maximal effect of optional stopping on $\alpha_{t}$ by attempting to maximize $E_{0}\left(Z_{t}\right)$ by choice of $t$.

We present numerical solutions to the optimal stopping problem in Section 2 for some special cases. The effect of optional stopping on the significance level is surprisingly small in the examples considered, an increase by less than a factor of two.

Patients may be measured individually, but treated in batches of different sizes. In many situations treating in batches could be a more economical way of treatment. When we consider this complication, from the data we obtain it seems that 'the size of the batch' has a modest effect.

In Section 3, we find an approximation to the hypothesis testing problem which does not depend on the distribution of the variable $X$ but only on the correlation coefficient $(\varrho)$ between $X$ and $Y$. The approximation is thus found when we formulate the analogous problem in continuous time.

\section{Numerical solutions}

\subsection{Introduction}

We consider a numerical solution using the method of backward induction. The examples contain a number of important special cases and provide motivation for the general theoretical approach in Section 3.

We calculate the maximal effect on the significance level for testing the mean of a response variable $Y(E(Y)=\theta)$ for a one sided null hypothesis $\mathrm{H}_{0}: \theta \leqslant \theta_{0}$. The stopping time considered is defined with respect to a covariate $X$.

\subsection{Hypothesis testing}

Let $H$ be the unknown common joint distribution of the pairs of observable covariate-response random variables $\left(X_{i}, Y_{i}\right), i=1,2, \ldots$ We suppose that $Y$ has an 
arbitrary distribution with unknown mean $\theta=E(Y)$, and finite variance $\sigma_{0}^{2}=V(Y)$ which is known under $\mathrm{H}_{0}$. We want to perform a one side sequential test for $\theta$, say $\mathrm{H}_{0}: \theta \leqslant \theta_{0}$.

Lemma 2.1. If the conditional expectation of $Y$ given $X$ is a linear function of $X$, then

$$
E\left(\bar{Y}_{n}-\theta \mid X_{1}, \ldots, X_{n}\right)=\delta\left(\bar{X}_{n}-\mu\right)
$$

and, if the conditional variance $\sigma^{2}$ of $Y$ given $X$ is independent of $X$, then

$$
V\left\{\bar{Y}_{n} \mid X_{1}, \ldots, X_{n}\right\}=\frac{\sigma^{2}}{n}
$$

where $\delta$ is a constant and $\mu=E(X)$.

In the remainder of this section, the covariate variable $X$ is assumed to be a dichotomous random variable taking the values 0 and 1 with probability $\frac{1}{2}$ each. Then, the conditional expectation of $Y$ given $X$ is linear, say $E(Y \mid X)=\delta X+\beta$. The conditional variance of $Y$ given $X$ is assumed to be independent of $X$, say $V(Y \mid X)=$ $\sigma^{2}<\infty$. By Lemma 2.1, the Central Limit Theorem suggests approximating $Z_{n}$ by

$$
Z_{n}^{*}=1-\Phi\left\{\frac{c \sigma_{0}-\delta \sqrt{n}\left(\bar{X}_{n}-\mu\right)}{\sigma}\right\}=1-\Phi\left\{\frac{c-\varrho \sqrt{n} \sigma_{x}^{-1}\left(\bar{X}_{n}-\mu\right)}{\sqrt{1-\varrho^{2}}}\right\}
$$

When $\varrho=0$ this reduces to the nominal value $1-\Phi(c)$. We solve the approximate problem, in which $Z_{n}$ is replaced by $Z_{n}^{*}$. That is, we find

$$
V^{*}=\max _{t \in C} E_{0}\left(Z_{t}^{*}\right)
$$

Observe that $Z_{n}^{*}=Z_{n}$ if the conditional distribution of $Y$ given $X$ is normal.

\subsection{The binomial example}

Consider the symmetric case in Table 1. Then $E(Y)=\theta, \operatorname{corr}(X, Y)=\varrho$ and $E(Y \mid X)=\theta+\varrho(X-\theta)$. For $\theta=\frac{1}{2}, V(Y \mid X)=\frac{1}{4}\left(1-\varrho^{2}\right)$ that is, the conditional variance of $Y$ given $X$ is independent of $X$, and the conditions of Lemma 2.1 are satisfied.

We may write the approximate payoff as

$$
Z_{n}^{*}=1-\Phi\left\{\frac{c-2 \sqrt{n} \varrho\left(\bar{X}_{n}-\frac{1}{2}\right)}{\sqrt{1-\varrho^{2}}}\right\}
$$

Table 1

\begin{tabular}{cccc}
\hline & $X=0$ & $X=1$ \\
\hline$Y=0$ & $(1-\theta)^{2}+\varrho \theta(1-\theta)$ & $\theta(1-\theta)-\varrho \theta(1-\theta)$ & $1-\theta$ \\
$Y=1$ & $\theta(1-\theta)-\varrho \theta(1-\theta)$ & $\theta^{2}+\varrho \theta(1-\theta)$ & $\theta$ \\
& $1-\theta$ & $\theta$ & \\
\hline
\end{tabular}




\subsection{Dynamic programming}

In this section we suppose that $X$ takes the values 0 and 1 with probability $\frac{1}{2}$ each and that the conditional variance of $Y$ given $X$ is independent of $X$. Then

$$
Z_{n}^{*}=1-\Phi\left\{\frac{c-2 \sqrt{n} \varrho\left(\bar{X}_{n}-\frac{1}{2}\right)}{\sqrt{1-\varrho^{2}}}\right\}=\psi_{n}\left(S_{n}\right), \text { say, }
$$

where $S_{n}=\sum_{i=1}^{n} X_{i}$.

Consider the restricted problem in which $t$ is required to be at most some specified $N$, and at least some specified $m$. The value for this restricted problem is

$$
V_{m}^{N}=\sup _{t \in C_{m}^{N}} E_{0}\left(Z_{i}^{*}\right)
$$

Table 2

\begin{tabular}{|c|c|c|c|}
\hline \multirow[t]{2}{*}{$m$} & \multicolumn{3}{|c|}{$N=25, c=1.96$} \\
\hline & $\varrho=0.2$ & $\varrho=0.4$ & $\varrho=0.6$ \\
\hline 1 & 0.0302563 & 0.0347522 & 0.0388983 \\
\hline 2 & 0.0298616 & 0.0347522 & 0.0388983 \\
\hline 3 & 0.0291243 & 0.0336786 & 0.0338983 \\
\hline 4 & 0.0286438 & 0.0325705 & 0.0374607 \\
\hline 5 & 0.0282968 & 0.0318539 & 0.0360676 \\
\hline 6 & 0.0279349 & 0.0311908 & 0.0351405 \\
\hline 7 & 0.02769 & 0.0305473 & 0.0342162 \\
\hline 8 & 0.02742 & 0.0300974 & 0.033292 \\
\hline 9 & 0.027203 & 0.029593 & 0.032616 \\
\hline 10 & 0.0269886 & 0.0291692 & 0.0318769 \\
\hline 11 & 0.0267989 & 0.0287644 & 0.0312053 \\
\hline 12 & 0.0266197 & 0.0283809 & 0.0306324 \\
\hline 13 & 0.0264521 & 0.0280448 & 0.0300252 \\
\hline 14 & 0.0262974 & 0.0277005 & 0.0295269 \\
\hline 15 & 0.0261482 & 0.0274142 & 0.0289993 \\
\hline 16 & 0.0260105 & 0.0271086 & 0.0285209 \\
\hline 17 & 0.0258765 & 0.0268451 & 0.0280584 \\
\hline 18 & 0.0257513 & 0.0265709 & 0.0276085 \\
\hline 19 & 0.0256298 & 0.0263258 & 0.0271958 \\
\hline 20 & 0.0255143 & 0.026076 & 0.0267767 \\
\hline 21 & 0.0254034 & 0.025848 & 0.0264001 \\
\hline 22 & 0.0252955 & 0.0256176 & 0.0260112 \\
\hline 23 & 0.0251935 & 0.0254053 & 0.0256582 \\
\hline 24 & 0.0250925 & 0.0251917 & 0.0252976 \\
\hline 25 & 0.0249974 & 0.0249896 & 0.0249551 \\
\hline Nominal & 0.0249979 & 0.0249979 & 0.0249979 \\
\hline Actual/nominal & 1.21 & 1.39 & 1.56 \\
\hline
\end{tabular}

Actual values for testing hypothesis (without batches) 
where

$$
C_{m}^{N}=\{t ; t \text { is a stopping rule, } m \leqslant t \leqslant N\} .
$$

To find the optimal solution for $V_{m}^{N}$ we use the theorem which can be found in Chow, Robbins and Siegmund (1971, p. 50).

Let $N$ be a fixed positive integer. Define successively $\gamma_{N}^{N}, \gamma_{N-1}^{N}, \ldots, \gamma_{m}^{N}$ by setting

$$
\begin{aligned}
& \gamma_{N}^{N}=Z_{N}^{*}=\psi_{N}\left(S_{N}\right), \\
& \gamma_{n}^{N}=\max \left(Z_{n}^{*}, E_{0}\left(\gamma_{n+1}^{N} \mid F_{n}\right)\right) \quad(n=N-1, \ldots, m) .
\end{aligned}
$$

For each $n=m, m+1, \ldots, N$ let

$$
s_{n}^{N}=\inf \left\{i \geqslant n, Z_{i}^{*}=\gamma_{i}^{N}\right\} \text {. }
$$

It follows from that theorem that $s_{m}^{N}$ is optimal in $C_{m}^{N}$.

In our problem, the recursion has a very simple form. Let $k$ denote a typical value of $S_{n}$, i.e., $k=0,1, \ldots, n$, and let $\gamma^{N}(N, k)=\psi_{N}(k)$ as defined in $(2.5)$. Then $\gamma_{n}^{N}=\gamma^{N}\left(n, S_{n}\right)$, where

$$
\gamma^{N}(n, k)=\max \left\{\psi_{n}(k), \frac{\gamma^{N}(n+1, k)+\gamma^{N}(n+1, k+1)}{2}\right\}
$$

for $m \leqslant n<N$.

It is straightforward to compute $\gamma^{N}(n, k)$ numerically for fixed $N$ and $m$.

Table 2 presents the actual values and the nominal values for different values of $\varrho, c, m$ and $N$. The effect of optimal stopping appears to be quite modest for the values of $N$ and $\varrho$ considered. For example, when $N=25, c=1.96$ and $\varrho=0.6$, the actual (worst case) value is $3.89 \%$, less than $1.5 \%$ larger than the nominal value of $2.49 \%$, and this is the largest increase reported in any of the tables. For $N=10$, $c=1.96$, and $\varrho=0.2$, the actual (worst case) value is $2.91 \%$, less than $0.5 \%$ larger than the nominal value of $2.49 \%$.

As expected, the effect increases with $N$ and $\varrho$. By the law of the iterated logarithm, the value must approach one as $N \rightarrow \infty$. The tables indicate that the limit is approached very slowly.

One possible explanation for the small increase is that the $Z_{n}$ are conditional probabilities while the nominal alpha is unconditional. Comparisons with a nominal alpha computed conditionally given the covariates awaits future work.

\subsection{Hypothesis testing: observations taken in batches}

It might not be feasible to analyze the data one at a time; instead we might need to see them in groups of size $M$, say. We will study the effect of batch size in this section.

Denote the reward by $G(k, j)$ where the sample size $n$ is $n=M k$ and $j$ is a typical value of $S_{n}$, i.e. $j=0,1, \ldots, M k$. Then, the recursion takes the form

$$
G(K, j)=v(K, j)=\psi_{M K}(j) \quad(j=0,1, \ldots, M K),
$$




$$
G(k, j)=\max \left\{v(k, j) ; \sum_{i=0}^{M}\left(\begin{array}{c}
M \\
i
\end{array}\right)\left(\frac{1}{2}\right)^{M} G(k+1, j+i)\right\}
$$

for $j=0, \ldots, M k$ and $k=1, \ldots, K-1$.

The stopping rule which maximizes this approximation is

$$
\tau=\inf \{n ; M \leqslant n \leqslant N, v(k, j)=G(k, j)\} .
$$

Tables 3 and 4 present the actual values and the nominal values for different values of batches' size and total amount of observations. The size of a batch appears to have very little influence.

Table 3

\begin{tabular}{|c|c|c|c|}
\hline \multirow[t]{2}{*}{$n$} & \multicolumn{3}{|c|}{$K=25, M=5, c=1.96$} \\
\hline & $\varrho=0.2$ & $\varrho=0.4$ & $\varrho=0.6$ \\
\hline 5 & 0.0310776 & 0.0373151 & 0.0447215 \\
\hline 10 & 0.0297829 & 0.0352482 & 0.0421819 \\
\hline 15 & 0.0291408 & 0.0339142 & 0.0399647 \\
\hline 20 & 0.0286684 & 0.0328846 & 0.0382302 \\
\hline 25 & 0.0282947 & 0.0320360 & 0.0368202 \\
\hline 30 & 0.027962 & 0.0313182 & 0.0356413 \\
\hline 35 & 0.0276746 & 0.0307106 & 0.0346493 \\
\hline 40 & 0.0274260 & 0.0301735 & 0.0337392 \\
\hline 45 & 0.0271943 & 0.0296778 & 0.0329047 \\
\hline 50 & 0.0269879 & 0.0292301 & 0.0321442 \\
\hline 55 & 0.0267951 & 0.0288250 & 0.0314633 \\
\hline 60 & 0.0266171 & 0.0284414 & 0.0308259 \\
\hline 65 & 0.0264512 & 0.0280886 & 0.0302222 \\
\hline 70 & 0.0262946 & 0.0277563 & 0.0296635 \\
\hline 75 & 0.0261483 & 0.0274406 & 0.0291385 \\
\hline 80 & 0.0260081 & 0.0271473 & 0.0286339 \\
\hline 85 & 0.0258768 & 0.0268639 & 0.0281617 \\
\hline 90 & 0.0257500 & 0.0265977 & 0.0277078 \\
\hline 95 & 0.0256298 & 0.0263399 & 0.0272713 \\
\hline 100 & 0.0255141 & 0.0260954 & 0.0268590 \\
\hline 105 & 0.0254022 & 0.0258576 & 0.0264553 \\
\hline 110 & 0.0252960 & 0.0256319 & 0.0260730 \\
\hline 115 & 0.0251925 & 0.0254116 & 0.0256980 \\
\hline 120 & 0.0250937 & 0.0252011 & 0.0253400 \\
\hline 125 & 0.0249977 & 0.0249961 & 0.0249893 \\
\hline Nominal & 0.0249979 & 0.0249979 & 0.0249979 \\
\hline $\begin{array}{l}\text { Actual/nominal } \\
(n=M)\end{array}$ & 1.24 & 1.49 & 1.789 \\
\hline
\end{tabular}

Actual values for testing hypothesis (with batches) 
Table 4

Actual values for testing hypothesis (with batches)

\begin{tabular}{llll}
\hline$n$ & \multicolumn{3}{c}{$k=10, M=10, c=1.96$} \\
\cline { 2 - 4 } & $\varrho=0.2$ & $\varrho=0.4$ & $\varrho=0.6$ \\
\hline 10 & 0.0292559 & 0.034177 & 0.0397833 \\
20 & 0.0282157 & 0.0318416 & 0.0363462 \\
30 & 0.0274905 & 0.0303149 & 0.0339215 \\
40 & 0.0269492 & 0.0291543 & 0.0319715 \\
50 & 0.0265096 & 0.0282175 & 0.0303981 \\
60 & 0.0261324 & 0.0274116 & 0.0290396 \\
70 & 0.025802 & 0.0267076 & 0.027881 \\
80 & 0.0255074 & 0.0260816 & 0.0268291 \\
90 & 0.0252417 & 0.0255155 & 0.0258703 \\
100 & 0.0249977 & 0.0249958 & 0.0249874 \\
& & & \\
Nominal & 0.0249979 & 0.0249979 & 0.0249979 \\
Actual/nominal & & & \\
$(n=M$ ) & 1.17 & 1.37 & 1.59 \\
\hline
\end{tabular}

\section{Theoretical solution to the hypothesis testing problem}

In Section 3.1 we prove that $Z_{n}-Z_{n}^{*} \rightarrow 0$ with probability one. In Section 3.3 we approximate the discrete problem with the corresponding continuous problem. In doing so, we find that the approximate solution depends only on the correlation coefficient $(\varrho)$ between $X$ and $Y$, and not on the distribution of $X$.

\subsection{Convergence of $Z_{n}-Z_{n}^{*}$ to zero with probability one}

Since $Z_{n}=P_{0}\left\{\sqrt{n}\left(\bar{Y}_{n}-\theta_{c}\right) / \sigma_{0}>c \mid X_{1}, \ldots, X_{n}\right\}$ we see that $1-Z_{n}$ is a random variable. In addition to that, we know that $Y_{1}, \ldots, Y_{n}$ are conditionally independent variables given $X_{1}, \ldots, X_{n}$ and that the conditional distribution of $Y_{i}$ given $X_{1}, \ldots, X_{n}$ is the same as the conditional distribution of $Y_{i}$ given $X_{i}$.

Let

$$
\begin{aligned}
& \theta\left(X_{i}\right)=E\left(Y_{i} \mid X_{i}\right), \quad \sigma^{2}\left(X_{i}\right)=V\left(Y_{i} \mid X_{i}\right), \\
& \varrho=\operatorname{corr}(X, Y), \quad \sigma_{x}^{2}=V(X), \quad s_{n}^{2}=\sum_{i=1}^{n} \sigma^{2}\left(X_{i}\right) .
\end{aligned}
$$

Then, by the law of large numbers,

$$
\frac{s_{n}^{2}}{n} \rightarrow \sigma^{2}=\int V(Y \mid X) \mathrm{d} F(x) \text { and } \sigma^{2}=\sigma_{0}^{2}\left(1-\varrho^{2}\right) .
$$

We see that

$$
\sum\left(Y_{i}-\theta\left(X_{i}\right)\right)=n\left(\bar{Y}_{n}-\theta_{0}\right)-\varrho \frac{\sigma_{0}}{\sigma_{x}} n\left(\bar{X}_{n}-\mu\right)
$$


and

$$
Z_{n}=P_{0}\left\{\frac{\sum_{i=1}^{n}\left(Y_{i}-\theta\left(X_{i}\right)\right)}{s_{n}}>\frac{\sigma_{0} \sqrt{n}}{s_{n}}\left[c-\frac{\varrho}{\sigma_{x}} \sqrt{n}\left(\bar{X}_{n}-\mu\right)\right] \mid X_{1}, \ldots, X_{n}\right\} .
$$

If we define

$$
G_{n}(z)=G_{n}\left(z ; X_{1}, \ldots, X_{n}\right)=P_{0}\left\{\frac{\sum_{i=1}^{n}\left(Y_{i}-\theta\left(X_{i}\right)\right)}{s_{n}} \leqslant z \mid X_{1}, \ldots, X_{n}\right\},
$$

then

$$
Z_{n}=1-G_{n}\left\{\frac{\sigma_{0} \sqrt{n}}{s_{n}}\left(c-\frac{\varrho}{\sigma_{x}} \sqrt{n}\left(\bar{X}_{n}-\mu\right)\right)\right\} .
$$

Theorem 3.1. $Z_{n}-Z_{n}^{*} \rightarrow 0$ with probability one.

Proof. We can write

$$
Z_{n}-Z_{n}^{*}=Z_{n}-\Phi_{n}\left\{-\sqrt{n}\left(X_{n}-\mu\right)\right\}-\left\{Z_{n}^{*}-\Phi_{n}\left\{-\sqrt{n}\left(\bar{X}_{n}-\mu\right)\right\}\right\}
$$

where

$$
\Phi_{n}(z)=1-\Phi\left\{\frac{\sigma_{0} \sqrt{n}}{s_{n}}\left(c+\frac{\varrho}{\sigma_{x}} z\right)\right\} .
$$

Part 1. We first prove that

$$
\left|G_{n}\left\{\frac{\sigma_{0} \sqrt{n}}{s_{n}}\left(c-\frac{\varrho \sqrt{n}}{\sigma_{x}}\left(\bar{X}_{n}-\mu\right)\right)\right\}-\Phi_{n}\left(-\sqrt{n}\left(\bar{X}_{n}-\mu\right)\right)\right| \rightarrow 0 \quad \text { w.p. } 1 .
$$

If we define

then

$$
Y_{n i}=\frac{Y_{i}-\theta\left(X_{i}\right)}{s_{n}}
$$

$$
S_{n}=S_{n n}=\sum_{i=1}^{n} Y_{n i}=\frac{\sum_{i=1}^{n}\left(Y_{i}-\theta\left(X_{i}\right)\right)}{s_{n}} .
$$

Thus, we are dealing with a double array of (conditionally) independent random variables:

$$
\begin{aligned}
& Y_{11} \\
& Y_{21}, Y_{22} ; \\
& Y_{31}, Y_{32}, Y_{33} ; \\
& \ldots \\
& Y_{n 1}, Y_{n 2}, \ldots, Y_{n n} ; \\
& \ldots
\end{aligned}
$$


for which

$$
\sum_{i=1}^{n} \sigma^{2}\left(Y_{n i}\right)=1
$$

The Central Limit Theorem asserts that $S_{n}$ converges in distribution to the unit normal distribution if Lindeberg's condition is satisfied.

Let

$$
L F_{n}(\varepsilon):=\frac{1}{s_{n}^{2}} \sum_{i=1}^{n} \int_{\left|y_{i}-\theta\left(X_{i}\right)\right| \geqslant \varepsilon s_{n}}\left|y_{i}-\theta\left(X_{i}\right)\right|^{2} \mathrm{~d} G_{i}\left(y_{i}\right) .
$$

If $L F_{n}(\varepsilon) \rightarrow 0$ for all $\varepsilon$, then $S_{n}$ converges in distribution to the standard normal distribution. Thus, we must show that $L F_{n}(\varepsilon) \rightarrow 0$ w.p. 1 for all $\varepsilon>0$.

Define the event

$$
B_{n}=\left\{s_{n}^{2} \geqslant \frac{1}{2} n A\right\} \quad \text { where } A=\sigma_{0}^{2}\left(1-\varrho^{2}\right) .
$$

Then, the indicator function of the complement of $B_{n}$

$$
I_{B_{n}^{\prime}} \rightarrow 0 \quad \text { w.p. } 1 \text {. }
$$

If $B_{n}$ occurs, then for all $c>0$ there exists $n_{0}$ such that for all $n \geqslant n_{0}$, we have

$$
L F_{n}(\varepsilon) I_{B_{n}} \leqslant \frac{2}{n A} \sum_{i=1}^{n} \int_{\left|y_{i}-\theta\left(X_{i}\right)\right| \geqslant c}\left|y_{i}-\theta\left(X_{i}\right)\right|^{2} \mathrm{~d} G_{i}\left(y_{i}\right) .
$$

If we define

$$
u_{c}\left(X_{i}\right)=\int_{\left|y_{i}-\theta\left(X_{i}\right)\right| \geqslant c}\left|y_{i}-\theta\left(X_{i}\right)\right|^{2} \mathrm{~d} G_{i}\left(y_{i}\right)
$$

we see that the random variables $u_{c}\left(X_{i}\right)$ are i.i.d.; so, applying the law of large numbers

$$
\frac{\sum_{i=1}^{n} u_{c}\left(X_{i}\right)}{n} \rightarrow \int_{-\infty}^{\infty} u_{c}(x) \mathrm{d} F(x)
$$

where

$$
\int_{-\infty}^{\infty} u_{c}(x) \mathrm{d} F(x)=\iint_{|y-\theta(x)|>c}|y-\theta(x)|^{2} \mathrm{~d} H(x, y)
$$

and $H$ denotes the joint distribution of $X$ and $Y$. Then, if $c \rightarrow \infty$,

$$
\iint_{|Y-\theta(x)| \geqslant c}|Y-\theta(x)|^{2} \mathrm{~d} H(x, y) \rightarrow 0 .
$$

The Lindeberg-Feller condition is satisficd w.p. 1 , so $G_{n}$ converges weakly to $\Phi$ w.p. 1 Pólya's theorem asserts: If $F_{n}$ and $F$ are distribution functions, and if $F_{n}$ converges weakly to $F$, and $F$ is continuous, then the convergence is uniform, i.e. $\sup _{x}\left|F_{n}(x)-F(x)\right| \rightarrow 0$. Using Pólya's theorem, we see that $G_{n}$ converges uniformly to $\Phi$. So 


$$
\begin{aligned}
Z_{n} & -\Phi_{n}\left(-\sqrt{n}\left(\bar{X}_{n}-\mu\right)\right) \\
& =G_{n}\left\{\frac{\sigma_{0} \sqrt{n}}{s_{n}}\left(c-\frac{\varrho \sqrt{n}}{\sigma_{x}}\left(\bar{X}_{n}-\mu\right)\right)\right\}-\Phi\left\{\frac{\sigma_{0} \sqrt{n}}{s_{n}}\left(c-\frac{\varrho \sqrt{n}}{\sigma_{x}}\left(\bar{X}_{n}-\mu\right)\right)\right\} \rightarrow 0 .
\end{aligned}
$$

Part 2. We now show that

$$
\Phi\left\{\frac{\sigma_{0} \sqrt{n}}{s_{n}}\left(c-\frac{\varrho \sqrt{n}}{\sigma_{x}}\left(\bar{X}_{n}-\mu\right)\right)\right\}-Z_{n}^{*} \rightarrow 0 .
$$

This follows easily from Pólya's theorem with

$$
F_{n}(z)=\Phi\left\{\frac{\sigma_{0}}{s_{n}} \sqrt{n}\left(c+\frac{\varrho}{\sigma_{x}} z\right)\right\} \text { and } F(z)=\Phi\left\{\frac{1}{\sqrt{1-\varrho^{2}}}\left(c+\frac{\varrho}{\sigma_{x}} z\right)\right\}
$$

for $-\infty<z<\infty$. Then $F_{n}$ converges weakly to $F$ w.p. 1 . So

$$
\begin{aligned}
\left|Z_{n}^{*}-F_{n}\left(-\sqrt{n}\left(\bar{X}_{n}-\mu\right)\right)\right| & =\left|F\left(-\sqrt{n}\left(\bar{X}_{n}-\mu\right)\right)-F_{n}\left(-\sqrt{n}\left(\bar{X}_{n}-\mu\right)\right)\right| \\
& \leqslant \sup _{z}\left|F_{n}(z)-F(z)\right| \rightarrow 0 .
\end{aligned}
$$

Theorem 3.2 will give us an approximation which does not depend on the distribution of $X$. We use the corresponding continuous problem.

\subsection{Approximate solution using the corresponding continuous problem}

Let $\{W(t)\}_{t \geqslant 0}$ be a normalized Brownian motion, then we will show that

$$
\sup _{m \leqslant \tau \leqslant N} E\left(Z_{\tau}^{*}\right)-\sup _{1 \leqslant \tau \leqslant 1 / \varepsilon} E\left\{1-\Phi\left\{\frac{c-\varrho W(\tau) / \sqrt{\tau}}{\sqrt{1-\varrho^{2}}}\right\}\right\} \rightarrow 0
$$

as $m, N \rightarrow \infty$ with $m / N \rightarrow \varepsilon$, where $Z_{n}^{*}$ was defined in (3.1) and the suprema extend over stopping times.

Let $W(t)$ be a normalized Wiener process, then

$$
W^{m}(t)=\sqrt{m} W\left(\frac{t}{m}\right)
$$

is also a Wiener process. For this $W^{m}(t)$ we use Skorokhod's representation theorem to construct stopping times $\sigma_{m 1}, \sigma_{m 2}, \ldots$ such that the partial sums

$$
S_{n} \stackrel{\text { D }}{=} W^{m}\left(\sigma_{m n}\right) \text {. }
$$

There is no loss of generality in supposing that $S_{n}$ is equal to $W^{m}\left(\sigma_{m n}\right)$ for all $n=1,2, \ldots$.

We define

We know that

$$
W_{m}(t)=\frac{S_{[m t]}}{\sqrt{m}}=\frac{1}{\sqrt{m}} W^{m}\left(\sigma_{m[m t]}\right)=W\left(\frac{\sigma_{m[m t]}}{m}\right) .
$$

$$
\sup _{0 \leqslant t \leqslant c}\left|W_{m}(t)-W(t)\right| \stackrel{\mathrm{P}}{\longrightarrow} 0 \quad \text { as } m \rightarrow \infty
$$


for any $c$. Now we define

$$
D_{m}(t)=\sigma\left\{W_{m}(s): s \leqslant t\right\}, \quad D(t)=\sigma\{W(s): s \leqslant t\}
$$

and let $C_{m}$ be the class of all $\tau$ s.t. $\tau \in[1,1 / \varepsilon]$ w.r.t. $D_{m}(t)$ and $C$ be the class of all $\tau$ s.t. $\tau \in[1,1 / \varepsilon]$ w.r.t. $D(t)$. Further, let

Then

$$
\begin{aligned}
& u(t, x)=1-\Phi\left\{\frac{c-\varrho x / \sqrt{t}}{\sqrt{1-\varrho^{2}}}\right\} . \\
& Z_{\tau}^{*}=u\left(\tau, W_{m}(\tau)\right) \text { and } u(\tau, W(\tau))=1-\Phi\left\{\frac{c-\varrho W(\tau) / \sqrt{\tau}}{\sqrt{1-\varrho^{2}}}\right\} .
\end{aligned}
$$

Theorem 3.2.

$$
\sup _{\tau \in C_{m}} E\left\{u\left(\tau, W_{m}(\tau)\right)\right\}-\sup _{\tau \in C} E\{u(\tau, W(t))\} \rightarrow 0
$$

as $m, N \rightarrow \infty$ with $m / N \rightarrow \varepsilon$.

Proof. With $W_{m}(\tau)$ as defined in (3.2) let $C_{m, k}$ be the class of all $\tau$ s.t. $\tau \in[1,1 / \varepsilon]$ w.r.t. $D_{m}(t)$, which are of the form $\tau=j / k, j=k, \ldots, k / \varepsilon$, and let $C_{k}$ be the class of all $\tau$ s.t. $\tau \in[1,1 / \varepsilon]$ w.r.t. $D(t)$, which are of the form $\tau=j / k, j=k, \ldots, k / \varepsilon$. Then

$$
\begin{array}{rl}
\sup _{\tau \in C_{m}} & E\left\{u\left(\tau, W_{m}(\tau)\right)\right\}-\sup _{\tau \in C} E\{u(\tau, W(\tau))\} \\
= & \sup _{\tau \in C_{m}} E\left\{u\left(\tau, W_{m}(\tau)\right)\right\}-\sup _{\tau \in C_{m, k}} E\left\{u\left(\tau, W_{m}(\tau)\right)\right\} \\
& +\sup _{\tau \in C_{m, k}} E\left\{u\left(\tau, W_{m}(\tau)\right)\right\}-\sup _{\tau \in C_{k}} E\{u(\tau, W(\tau))\} \\
& +\sup _{\tau \in C_{k}} E\{u(\tau, W(\tau))\}-\sup _{\tau \in C} E\{u(\tau, W(\tau))\} \\
= & I+\mathrm{II}+\mathrm{III} .
\end{array}
$$

Next we prove that III $\rightarrow 0$. If $\tau$ is a stopping time, let

$$
\tau_{k}=\frac{[(k+1) \tau]}{k} \wedge \frac{1}{\varepsilon}
$$

Then $\tau_{k} \in C_{k}\left(\tau_{k} \leqslant t\right.$ iff $k \tau_{k} \leqslant k t$ iff $(k+1) \tau \leqslant[k t]$ which implies that $\left.\tau_{k} \in C_{k}\right)$ and $\left|\tau_{k}-\tau\right| \leqslant 1 / k$. It follows easily that III $\rightarrow 0$ as $k \rightarrow \infty$ and a similar argument shows that $\sup _{\tau} \mathrm{I} \rightarrow 0$.

For a fixed $k$, we can use the backward induction technique of dynamic programming to obtain

$$
\lim _{m \rightarrow \infty} \operatorname{II}(m, k)=0 \quad \text { for all } k \text {. }
$$

To see why, fix $K$, let $K=k / \varepsilon$, and define functions $u_{j}^{m}$ and $v_{j}, j=k, \ldots, K$, by

$$
u_{K}^{m}(y)=v_{K}(y)=u\left(\frac{K}{k}, y\right)
$$


and

$$
u_{j}^{m}(y)=\max \left\{u\left(\frac{j}{k}, y\right), \quad \int u_{j+1}^{m}(y+z) \mathrm{d} F_{m, j}(z)\right\}
$$

$$
v_{j}(y)=\max \left\{u\left(\frac{j}{k}, y\right), \quad \int v_{j+1}(y+z) \mathrm{d} \Phi_{k}(z)\right\}
$$

for $-\infty<y<\infty, j=k, \ldots, K-1$, where

and

$$
\Phi_{k}(z)=\Phi(z \sqrt{k}), \quad F_{m, j}(z)=P\left(S_{m j} \leqslant z \sqrt{m}\right)
$$

$$
m_{j}=\left[\frac{m(j+1)}{k}\right]-\left[\frac{m j}{k}\right]
$$

for $-\infty<z<\infty$ and $j=k, \ldots, K-1$. Then

and

$$
\sup _{\tau \in C_{m, k}} E\left\{u\left(\tau, W_{m}(\tau)\right)\right\}=E\left\{u_{k}^{m}\left(W_{m}(1)\right)\right\}
$$

$$
\sup _{\tau \in C_{k}} E\{u(\tau, W(\tau))\}=E\left\{v_{k}(W(1))\right\}
$$

by the dynamic programming relations. Since $W_{m}(1)$ converges in distribution to $W(1)$ as $m \rightarrow \infty$, it suffices to show that

$$
u_{k}^{m}(y) \rightarrow v_{k}(y)
$$

uniformly on compacts (in $y$ ). This may be done by backward induction. If $j=K$, then $u_{j}^{m}(y)=u(K / k, y)=v_{j}(y)$ for all $m$. So the relation is obvious. Now suppose that

$$
u_{i}^{m}(y) \rightarrow v_{i}(y)
$$

uniformly on compacts in $y$ as $m \rightarrow \infty$ for all $i>j$, where $k \leqslant j<K$. Then, since $F_{m, j}$ converges weakly to $\Phi_{k}$,

$$
\begin{aligned}
u_{j}^{m}(y) & =\max \left\{u\left(\frac{j}{k}, y\right), \int_{-\infty}^{\infty} u_{j+1}^{m}(y+z) \mathrm{d} F_{m, j}(z)\right\} \\
& \rightarrow \max \left\{u\left(\frac{j}{k}, y\right), \int_{-\infty}^{\infty} v_{j+1}(y+z) \mathrm{d} \Phi_{k}(z)\right\} \\
& =v_{j}(z)
\end{aligned}
$$

as $m \rightarrow \infty$. So the induction is complete and (4.4) follows. This completes the proof that $\mathrm{II} \rightarrow 0$ and therefore the proof of the theorem.

\section{References}

Abramowitz, M. and A. Stegun (1964). Handbook of Mathematical Tables. National Bureau of Standards, Washington, D.C. 
Billingsley, P. (1968). Convergence of Probability Measures. John Wiley, New York. Breiman, L. (1968). Probability. Addison-Wesley, Reading, MA.

Chung, K.L. (1974). A Course in Probability Theory. Academic Press, New York.

Chow, Y., H. Robbins and D. Siegmund (1971). Great Expectations: The Theory of Optimal Stopping. Houghton Mifflin, Boston, MA.

Freeman, D. (1983). Brownian Motion and Diffusion. Springer, New York.

Siegmund, D. (1985). Sequential Analysis. Test and Confidence Intervals. Springer, New York. 